\title{
The Heart has Intrinsic Ketogenic Capacity that is Facultatively Upregulated in Heart Failure with Preserved Ejection Fraction
}

John O'Sullivan ( $\nabla$ john.osullivan@hri.org.au )

The University of Sydney

\section{Yen Chin Koay}

The University of Sydney

\section{Yang Cao}

University of California Los Angeles

\section{Angela Yu Bai}

The University of Sydney

\section{Sean Lal}

University of Sydney https://orcid.org/0000-0002-5841-560X

\author{
Aldons Lusis \\ University of California Los Angeles https://orcid.org/0000-0001-9013-0228 \\ David Kaye \\ Baker IDI Heart and Diabetes Institute

\section{Mark Larance} \\ Charles Perkins Centre, School of Life and Environmental Sciences, University of Sydney \\ https://orcid.org/0000-0002-8579-2267
}

\section{Biological Sciences - Article}

Keywords: heart failure, ejection fraction, therapeutic strategies

Posted Date: June 22nd, 2021

DOl: https://doi.org/10.21203/rs.3.rs-607376/v1

License: (c) (i) This work is licensed under a Creative Commons Attribution 4.0 International License. Read Full License 


\section{Abstract}

Heart Failure with preserved Ejection Fraction or 'HFpEF' is now the most common type of heart failure worldwide, with considerable morbidity and mortality, and with no effective pharmacotherapies. For the first time, we show that the human and murine HFpEF heart have impaired uptake of ketone bodies, and reveal an intrinsic capacity of human and murine hearts to generate their own ketones via the canonical rate-limiting ketogenic enzyme, 3-hydroxy-3-methylglutaryl CoA synthase 2 (HMGCS2). We demonstrate that protein levels of HMGCS2 are dramatically elevated in HFpEF myocardium that does not overcome reduced specific activity of this enzyme. This appeared to be due to hyperacetylation caused by depletion of the de-acetylating enzyme sirtuin 3 and decreased oxidized/reduced nicotinamide adenine dinucleotide (NAD+/NADH) ratio, upon which the deacetylation activity of sirtuins is dependent. Also for the first time, we show that the canonical stimuli that serve to turn on ketogenesis in the liver are present locally in the HFpEF heart, augmented by a profound energy deficiency as determined by depleted phosphocreatine/ adenosine triphosphate (PCr/ATP) ratio. Thus, metabolically inflexible HFpEF hearts try to auto-regulate their energy supply by upregulating ketogenesis as an apparent "rescue strategy". These results serve to reframe our understanding of cardiac ketone metabolism and open opportunities for novel therapeutic strategies for HFpEF, urgently needed.

\section{Introduction}

Of all organs, the heart has the highest energy requirements and resting metabolic rate per unit mass $(440 \mathrm{kcal} / \mathrm{kg} / \mathrm{day})^{2}$, and highest oxidative demand ${ }^{3}$. Therefore, it is critical that the heart can remain flexible in its choice of substrate in the face of changing nutrient supply, metabolic stress, and pathological injury. Certain states, such as diabetes and heart failure, impair this flexibility. In these states, alternative substrates such as ketone bodies become extremely important. It is thought that ketone bodies are a favoured substrate under conditions of stress and/or metabolic inflexibility as they are a "thrifty" substrate ${ }^{4}$; compared to fatty acids, ketones are more energetically efficient, yielding more energy per unit cost in oxygen consumption ( $\mathrm{P} / \mathrm{O}$ ratio) ${ }^{5-7}$. Recently, both clinical ${ }^{8}$ and preclinical ${ }^{9}$ data demonstrated beneficial effects of ketone administration in heart failure, and in HFpEF specifically ${ }^{10}$.

Mechanistic study of HFpEF has been challenging due to the heterogeneity of clinical presentation, poor diagnostic criteria, and the absence of clinically-representative models. Recently, it was shown that invasive haemodynamic assessment during exercise provides superior diagnostic ${ }^{11}$ and predictive $e^{12}$ classification of HFpEF. Furthermore, a recent murine model was developed that is highly-representative of the human HFpEF syndrome ${ }^{13}$. Therefore, our goals were to: 1 ) use this murine model to address fundamental questions about metabolic flexibility and the role of myocardial ketone utilisation in HFpEF; 2) examine ketone metabolism in human myocardium; and 3) determine cardiac ketone uptake in carefully-defined human HFpEF that incorporates invasive exercise haemodynamic assessment.

\section{Results}




\section{HFpEF hearts have suppressed glycolysis and upregulated PDK4 causing metabolic inflexibility}

Compared to mice fed chow, mice fed a combination of high-fat diet (HFD) and L- $N^{G}$-Nitro Arginine Methyl Ester (L-NAME) for 5 weeks (HFpEF mice) showed significant increases in fat mass, impaired glucose tolerance, and insulin resistance as indicated by the measurement of glucose area under the curve (AUC) and homeostatic model assessment of insulin resistance (HOMA-IR) (Fig. 1a-c). HFpEF mice displayed cardiac hypertrophy by increased heart-weight-to-tibial-length ratio (Fig. 1d); elevated LV filling pressures by transmitral and tissue doppler (Fig. 1e,f); impaired global longitudinal strain (Fig.1g); and pulmonary congestion by increased wet/dry lung weight ratio (Fig. 1h). HFpEF myocardium had decreased levels of glucose transporters along with depleted glycolytic and glucose oxidative substrates, consistent with an insulin resistant heart (Fig. 1i). There were decreased protein abundances of several rate-limiting enzymes critical for the regulation of glucose uptake, glucose phosphorylation, and the conversion of pyruvate to acetyl-CoA (Fig. 1j, k). Levels of the insulin-responsive glucose transporter that regulates glucose entry in the beating heart, glucose transporter type 4 [GLUT4; also known as solute carrier family $2(\mathrm{SLC} 2 \mathrm{~A} 4)]^{14}\left(\mathrm{FC}=0.80, \mathrm{p}=6.5 \times 10^{-3}\right)$, and downstream hexokinase 1 and $2(\mathrm{HK} 1, \mathrm{FC}=$ $0.75, p=9.1 \times 10^{-3}$ and HK2, FC $\left.=0.69, p=4.7 \times 10^{-4}\right)$, were significantly decreased in HFpEF myocardium (Fig. 1j). HFpEF hearts had over 2.5-fold increase in pyruvate dehydrogenase kinase 4 (PDK4) expression $\left(F C=2.60, p=2.3 \times 10^{-5}\right)$, which can reduce glucose oxidation by deactivating the pyruvate dehydrogenase complex (PDHC), a key mitochondrial enzyme that catalyzes the oxidative decarboxylation of pyruvate to acetyl-CoA, linking glycolysis to the tricarboxylic acid cycle ${ }^{15}$. Two subunits of pyruvate dehydrogenase (E1), PDHA1 and PDHB, were significantly decreased (PDHA1, FC = $0.80, p=3.0 \times 10^{-2}$ and PDHB, FC = 0.83, $p=4.5 \times 10^{-2}$ ) in HFpEF myocardium. Glucose-6-phosphate (an important intermediate of glucose metabolism) was significantly decreased $\left(F C=0.71, p=9.3 \times 10^{-3}\right)$ in the hearts of HFpEF mice, consistent with the reduction of glucose transport into these hearts.

\section{Altered mitochondrial fatty acid oxidation, ketogenesis and ketone oxidation in HFpEF Myocardium}

As illustrated in the volcano plot in Fig. 2a, mitochondrial HMGCS2 changed far more than any other protein, with a 16-fold upregulation in HFpEF myocardium. HFpEF mice had significantly reduced levels of the myocardial ketone transporter monocarboxylate transporter 1 (MCT1, also called SLC16A1) (FC = $0.80, p=0.05$ ) (Fig. 2b, d) compared to control mice. Protein abundance of mitochondrial pyruvate carrier (MPC), which is used to transport ketone bodies into mitochondria, was found to be lower in HFpEF hearts. Despite decreased MPC protein levels in HFpEF hearts, there were significantly elevated levels of proteins that facilitate the uptake of fatty acids into the heart including fatty acid translocase (CD36) (FC $=1.2, \mathrm{p}=4.9 \times 10^{-3}$ ) and fatty acid transporters carnitine palmitoyltransferases 1 and 2 (CPT1 and CPT2) (CPT1, FC $=1.4, p=1.2 \times 10^{-2}$ and CPT2, FC $=1.3, p=5 \times 10^{-2}$ ). Acyl-CoA dehydrogenases (ACADs) catalyze the first step in mitochondrial $\beta$-oxidation of fatty acids and are classified according to substrate chain-length specificity for FAO: short chain acyl-CoA dehydrogenases (SCAD) (C4-C6), medium (MCAD) (C4-C12), long (LCAD) (C8-C20), and very long (VLCAD) (C12-C24). We found that protein abundance of VLCAD was significantly higher in HFpEF hearts (Extended Data Fig. 1a). The HFpEF heart produced 
energy almost exclusively from fatty acid oxidation, supported by a significant increase in medium- and long-chain acylcarnitine concentrations (Extended Data Fig. 1b).

In addition to elevated CPT1, CPT2 and ACADs, two key mitochondrial fatty acid oxidation enzymes, acylCoA thioesterase 2 (ACOT2) ( $F C=1.6, p=4.0 \times 10^{-3}$ ) and acetyl-coenzyme acyltransferase 2 (ACAA2) (FC $=1.1, p=4 \times 10^{-2}$ ) were also significantly increased. L-Carnitine, used for the transport of long-chain fatty acids into mitochondria for $\beta$-oxidation and consumed during increased fatty acid import for oxidation ${ }^{16}$, was significantly reduced $\left(F C=0.86, p=1 \times 10^{-2}\right)$ in HFpEF myocardium. Together, these findings suggest increased fatty acid uptake, mitochondrial transport, and oxidation within the HFpEF myocardium.

Characteristic of a rate-limiting enzyme, HMGCS2 served as a transition point, whereby upstream enzymes were increased and downstream enzymes unchanged or decreased (Fig. 2 b,d). Levels of the next enzyme in the cascade, HMG-CoA lyase (HMGCL), which catalyses the cleavage of HMG-CoA to liberate acetyl-CoA and acetoacetate (AcAc), did not change significantly in HFpEF myocardium. Further downstream, mitochondrial matrix enzyme 3-oxoacid CoA-transferase 1 (OXCT1) (FC = 0.66, $p=4.7 \times 10^{-}$ $\left.{ }^{4}\right)$ and beta-hydroxybutyrate dehydrogenase $1(\mathrm{BDH} 1)\left(\mathrm{FC}=0.47, \mathrm{p}=2.4 \times 10^{-2}\right)$, each involved in ketone body utilization, were both significantly decreased. Cardiac levels of ketone body acetoacetate (AcAC) (FC $\left.=0.88, p=3 \times 10^{-3}\right)$, but not beta-hydroxybutyrate $(\beta \mathrm{HB})$, was significantly lower in HFpEF mice. AcetylCoA, a metabolite at the intersection of many metabolic pathways and a sensitive indicator of perturbed metabolism, was found to be significantly reduced by $\sim 2$-fold $\left(p=1.5 \times 10^{-2}\right)$ in HFpEF myocardium. HFpEF hearts were energy-starved, as evidenced by a significant reduction in myocardial phosphocreatine-to-ATP ratio $(\mathrm{PCr} / \mathrm{ATP})\left(\mathrm{FC}=-2.93, \mathrm{p}=1.9 \times 10^{-3}\right)$, suggesting an energetically-deficient state $^{17,18}$.

To isolate the contribution of high-fat diet (without L-NAME) to changes in ketogenesis and ketolysis, we used high-fat diet only compared to chow. High-fat diets alone did not significantly change myocardial HMGCS2, HMGCL, BDH1, or OXCT1 levels (Extended Data Fig. 2a). We also observed no change in the $\beta \mathrm{Hb}$-to-AcAc ratio of the HFD hearts when compared to the control mice hearts (Extended Data Fig. 2b), while HFpEF mouse myocardium showed a significant increase in $\beta \mathrm{Hb}$-to-AcAc ratio (Extended Data Fig. 2c).

\section{Transcardiac gradients in human HFpEF reveal decreased ketone uptake compared to healthy controls, with a predilection for AcAC uptake compared to $\beta H B$}

In a cohort of HFpEF patients and controls (Extended Data Table 1), we determined transcardiac ketone body gradients. All patients underwent invasive haemodynamic assessment before and during exercise. As in Fig. $2 \mathrm{e}$ and $\mathrm{f}$, the ratio of percentage cardiac uptake (Arterial - coronary sinus concentration/ arterial concentration * 100 ) of both $B \mathrm{HB}$ and AcAc to pulmonary capillary wedge pressure was significantly decreased in HFpEF patients compared to controls, indicating impaired ketone transport in HFpEF, consistent with that seen in the HFpEF murine model (Fig. 2b,c). Further, comparing percent uptake of 
$\beta \mathrm{HB}$ with AcAc revealed a predilection for AcAC uptake compared to $\beta \mathrm{HB}$. In murine HFpEF myocardium, there was an increased $\beta \mathrm{HB} / \mathrm{AcAc}$ ratio (Extended Data Fig. $2 \mathrm{c}$ ), and this is consistent with increased AcAc compared to $\beta$ HB uptake in human HFpEF hearts.

\section{Murine and Human Hearts Have Intrinsic Ketogenic Capacity}

As described above, comprehensive proteomic profiling revealed that 3-hydroxy-3-methylglutaryl CoA synthase 2 (HMGCS2), the canonical rate-limiting ketogenic enzyme, was 16-fold elevated in HFpEF myocardium (Fig. 2a). We wanted to establish definitively whether the heart is capable of synthesizing ketone bodies via this canonical enzyme, which we assessed by condensation of $\beta$-oxidationderived $\left[1,2,3,4-{ }^{13} C_{4}\right]$ acetoacetyl-CoA and $\left[1,2-{ }^{13} C_{2}\right]$ acetyl-CoA to generate $\left[1,2,3,4,5,6-{ }^{13} C_{6}\right] \beta$-hydroxy $\beta$ methylglutaryl-CoA (HMG-CoA) catalyzed by HMGCS2, which is cleaved by HMG-CoA lyase (HMGCL) to generate $\left[1,2,3,4-{ }^{13} \mathrm{C}_{4}\right]$ acetoacetate. As illustrated in Fig. 3a, in both human and murine myocardium, ketogenesis is indeed an active process, with a time-dependent ${ }^{13} \mathrm{C}$ enrichment of HMGCS2 ketogenic intermediate HMG-CoA and product acetoacetate. As in Fig.3b, there was generation of ${ }^{13} \mathrm{C}$-acetoacetate at $2-3 \mathrm{nmol} / \mathrm{mg}$ protein.

\section{HMGCS2 specific activity is significantly impaired in HFpEF myocardium, which is not overcome by upregulation of protein levels}

Western blotting confirmed (Fig. 4a) the dramatic upregulation of HMGCS2 protein levels seen by proteomics (Fig. 3a). The overall activity of the ketogenic pathway, indicated by the rate at which $[1,2,3,4-$ ${ }^{13} \mathrm{C}_{4}$ lacetoacetate formed over time, however, did not change (Fig. 4a). Immunoprecipitation followed by western blotting revealed increased acetylation of lysine residues on HMGCS2 (Fig. 4b). This was accompanied by decreased Sirtuin $3\left(F C=0.76, p=9.3 \times 10^{-4}\right)$ levels in HFpEF myocardium (Fig. 4c). As reported in the liver, under normal conditions sufficient levels of Sirtuin 3 deacetylate HMGCS2 to maintain its active site in the correct conformation for binding substrate acetoacetyl-CoA, i.e. maintaining its ketogenic activity ${ }^{19}$. Sirtuin 3 is dependent on preserved NAD ${ }^{+} / N_{A D H}$ ratio ${ }^{20}$, which we found to be significantly decreased (Fig. 4c). Therefore, our results suggest that depleted NAD ${ }^{+} / \mathrm{NADH}$ leads to reduced Sirtuin 3 activity, resulting in over-acetylated HMGCS2 that is dysfunctional (Fig. 4d). We believe that this, in conjunction with the metabolic inflexibility reported above, served as a stimulus to upregulate HMGCS2 protein levels in the HFpEF myocardium.

\section{Discussion}

It was reported several decades ago that in the liver conditions of fatty acid over-supply along with reduced oxaloacetate (due to suppressed glucose metabolism) serve as a ketogenic stimulus, leading to acetyl-coA entering the ketogenic pathway ${ }^{21}$. Remarkably, we see the exact same conditions in HFpEF myocardium (Fig. 3b, c), reaffirming the same canonical generative pathway in the heart. Further, depletion of PCr/ATP confirms a state of significant energy depletion in the heart ${ }^{22}$, augmenting the 
stimulus to seek other sources of energy, and ketones are a preferred alternative energy source of the heart ${ }^{23}$. The current paradigm suggests that robust HMGCS2 expression only occurs in hepatocytes and gut epithelial cells: extra-hepatic tissues don't contribute ketone bodies to the circulation and they internalise ketones down a concentration gradient via MCT1/2-dependent mechanisms ${ }^{24,25}$. This includes the heart oxidising ketone bodies in direct proportion to their delivery, and the myocardium is the highest ketone body consumer per unit mass ${ }^{26-31}$. However, we observed decreased myocardial ketone body uptake from the circulation, whilst concurrently the levels of the rate-limiting ketogenic enzyme were dramatically upregulated.

It has been suggested that "apparent" extra-hepatic ketogenesis can occur in the context of decreased ketone oxidation/ consumption ${ }^{24}$, whereby decreased ketone oxidation leads to ketone body accumulation and gives the appearance of de novo ketogenesis. However, we did not see increased ketone body accumulation. Several extra-hepatic non-HMGCS2 "pseudoketogenic" processes have been described that include reversible enzymatic activity of thiolase and OXCT $1^{32}$. However, our data confirms bone fide canonical HMGCS2 ketogenesis in the heart. Regardless, we are not proposing that cardiac ketogenesis is important outside the heart or contributes to circulating ketones. Rather, our data demonstrates an unknown capacity of the heart to generate its own ketones at a time of metabolic inflexibility and critical fuel deprivation.

Previous studies of rodent Type 1 diabetes models reported upregulation of myocardial mRNA levels of HMGCS2 in mice ${ }^{33}$, and both its mRNA and protein levels in rats ${ }^{34}$. However, these studies did not determine enzymatic activity, post translational modification, or the relative changes in fatty acid oxidation, glucose metabolism, or citric acid cycle changes at the metabolite level, essential to pinpoint the transition to ketogenesis. Our assessment of HMGCS2 activity was critical for determining the actual state of myocardial ketogenesis. Measuring only mRNA or protein levels would have provided a profoundly misleading conclusion. Activity of HMGCS2 is dependent on continual deacetylation of HMGCS2 at several lysine residues. Sirtuin 3 is the major deacetylating enzyme, itself dependent on the $\mathrm{NAD}^{+} / \mathrm{NADH}$ ratio ${ }^{20,35}$. In addition to its role deacetylating HMGCS2, there are other roles of Sirtuin 3 that are important to consider in the context of heart failure and HFpEF, including maintaining mitochondrial function $^{36-38}$ and preventing cardiac hypertrophy ${ }^{36}$.

Impaired cardiac ketone uptake in the murine model was supported by our data in humans. Exercise pulmonary capillary wedge pressure (PCWP) has been shown to be a more accurate diagnostic criterion for $\mathrm{HFpEF}^{11,12}$. To our knowledge, we are the first to compare PCWP to transcardiac ketone gradients in $\mathrm{HFpEF}$ and controls. Using transcardiac gradients, others have shown that cardiac ketone uptake is increased in $\mathrm{HFrEF}$ compared to $\mathrm{HFpEF}^{39}$ and in $\mathrm{HFrEF}$ and aortic stenosis patients compared to controls $^{40}$. However, our data demonstrates decreased cardiac extraction of ketone bodies with elevations in exercise PCWP, a more accurate measure of HFpEF. This is an important finding, and is consistent with impaired ketone uptake in our murine HFpEF myocardium. 
HFpEF hearts favoured AcAc over $\beta H B$ uptake. In our murine model, there was depletion of AcAc but not $\beta \mathrm{HB}$ in murine HFpEF myocardium. As ketone uptake is reported to occur along circulating vs internal gradients via the MCT1/2 transporters, these findings are consistent. Indeed, our HFpEF murine myocardium showed a decreased expression of key ketolytic enzymes BDH1 and succinyl-coA:3-ketoacid CoA transferase (SCOT/OXCT1), accompanied by increased myocardial $\beta H B$-to-AcAc ratio. AcAc- $\beta H B$ redox coupling is important for maintaining myocardial energy homeostasis, and our data suggests uncoupling via BDH1 suppression, leading to depleted AcAc. This may serve as a further stimulus for ketogenesis to generate sufficient AcAc to supply acetyl-coA for the Krebs cycle.

Much recent work has focussed on the salutary effects of ketone bodies in heart failure-10,23,41. Exogenous ketone bodies are cardioprotective in HFpEF, allowing the heart to extricate energy at a lower oxygen cost ${ }^{42}$, and suppressing myocardial inflammation ${ }^{10}$. As such, local generation of ketones by the heart would serve as a valuable therapeutic target for multiple cardiac diseases. Herein, we provide data that challenges the current paradigm of the heart as only a consumer of ketones provided by the liver. We definitively demonstrate that both murine and human hearts are capable of ketogenesis via the canonical ketogenic enzyme. For the first time, we reveal that human HFpEF hearts have impaired ketone uptake. We show that in HFpEF, myocardial metabolic inflexibility leads to decreased glycolysis along with upregulated fatty acid oxidation in the presence of depleted oxaloacetate, serving as a stimulus to potently upregulate the canonical ketogenic enzyme in an apparent strategy to rescue energy deprivation. We believe these results reframe our understanding of cardiac substrate utilisation and offer a novel potential therapeutic strategy in HFpEF: targeting the heart's inherent ketogenic capacity.

\section{Methods}

\section{Animals and husbandry}

All procedures involving animals received ethics approval from the Institutional Animal Ethics Committee at the University of Sydney. Twenty male 5-week-old wild-type C57BL/6 mice were obtained from The Australian BioResources Pty Ltd facility in Moss Vale, New South Wales. Mice were housed and maintained on a 12-hour light-dark cycle with free access to water and food. Two weeks after arrival, mouse cages were randomly assigned as control or treatment group. While mice in the control group received a normal CHOW diet (SF14-162, Specialty Feeds Pty Ltd), mice in the treatment group were fed a combination of HFD (60\% calories from lard, SF18-072, Specialty Feeds Pty Ltd) and L-NAME (0.5 g/L in drinking water). After five weeks of different dietary regimens, all 20 mice were sacrificed using overdosed intraperitoneal injections of pentobarbital (Nembutal ${ }^{\circledR}$, Abbott Laboratories, North Chicago, IL, USA; 25 $\mathrm{mg} / \mathrm{mL}, 75 \mathrm{mg} / \mathrm{kg}$ ) as approved by the Institutional Animal Ethics Committee at the University of Sydney. Blood was collected via cardiac puncture using EDTA-coated syringes and sample tubes, then centrifuged to isolate plasma. Aliquots of plasma were frozen on dry ice and stored at $-80^{\circ} \mathrm{C}$ until analysis. The hearts were obtained, weighed and then clamped in liquid nitrogen stored metal clamps, flash frozen in liquid nitrogen and stored at $-80^{\circ} \mathrm{C}$ until use. 


\section{Anesthesia for mice echocardiography}

Echocardiography was performed in anaesthetised mice. Anaesthesia was commenced using 2-3\% isoflurane in oxygen in an induction chamber. Anaesthesia was maintained during imaging using $1-2 \%$ isoflurane in oxygen delivered via nose cone. During imaging, heart rate was maintained between 450550 beats per minute (bpm) and body temperature was kept at $36-37^{\circ} \mathrm{C}$ (rectal probe). The mice were made to lie on a heated imaging platform on their ventral side. All animal studies were approved by the Institutional Animal Ethics Committee at the University of Sydney.

\section{Echocardiography measurements}

A Vevo2100 system high-resolution ultrasound system with a 40-MHz linear probe (FUJIFILM VisualSonics Inc., Canada) was used to perform echocardiography on mice 5 weeks after commencement of diets. The echocardiographic measurements were performed according to a standard method published previously ${ }^{43}$. Several echocardiographic parameters including transmitral Doppler indices, Tissue Doppler, left atria area, and speckle training were used to assess diastolic function in mice. Echocardiographic measures of diastolic function including deceleration time (DT), peak early diastolic velocity $(E)$, peak late diastolic velocity $(A)$, peak early $\left(e^{\prime}\right)$ diastolic mitral annular velocity, and isovolumetric relaxation time (IVRT) were recorded. Left ventricular wall thickness including interventricular septal wall thickness (IVST), left ventricular posterior wall thickness (LVPWT), left ventricular end-diastolic diameter (LVEDd), left ventricular end-systolic diameter (LVESD), and left ventricular ejection fraction (LVEF) were also measured. All acquired images were digitally stored and assessed offline in blinded fashion using a dedicated VisualSonics workstation.

\section{Body composition}

Longitudinal measures of body composition were assessed using an EchoMRI 900 (EchoMRI, TX, USA) prior to culling at each experimental endpoint.

\section{Blood glucose and insulin measurement}

Oral Glucose Tolerance Tests (oGTT) were performed via oral gavage $(2 \mathrm{~g} / \mathrm{kg}$ lean mass) after 6 hours fasting. Basal blood glucose was measured through tail vein bleeding using a clinical glucometer (AccuChek Performa, Roche Diagnostics Australia Pty Ltd). Blood glucose was measured 15, 30, 45, 60, and 90 mins after glucose administration. Blood insulin levels were determined by the Ultra Sensitive Mouse Insulin ELISA kit (Crystal Chem Inc). Area under the curve (AUC) was derived from an oral glucose tolerance test, which indicates the time taken to clear a bolus dose of glucose from the bloodstream and return to basal levels. The Homeostasis Model Assessment of Insulin Resistance (HOMA-IR) was used as an estimation for global IR for each group. It was calculated by multiplying the fasting plasma insulin $(\mu \mathrm{U} / \mathrm{L})$ and fasting plasma glucose $(\mathrm{mM})$ and divided by constant $66^{44,45}$.

\section{Human heart tissue}


Donor left ventricular (LV) myocardium was obtained from donors whose hearts did not proceed to transplantation due to transportation logistics, donor-recipient mismatch in size, and immune

incompatibility, as previously described ${ }^{46-48}$. Histological analysis of the donor samples were demonstrated to be structurally normal as per formal pathological examination ${ }^{46}$. LV samples from the donors were collected and snap frozen $\left(-196^{\circ} \mathrm{C}\right)$ and were stored in the Sydney Heart Bank at -170 to $-180^{\circ} \mathrm{C}$ from the time of harvest ${ }^{46}$. Written consent was obtained to use tissue specimens for research. The study was approved by the Ethics Committee of The University of Sydney (USYD \#2016/923 \& 2020/ ETH01161) and was conducted in accordance with the Declaration of Helsinki.

\section{Invasive Exercise Haemodynamics and Transcardiac Gradients}

HFpEF patients were referred to the Department of Cardiology, Alfred Hospital for investigation of symptoms consistent with a diagnosis of heart failure (NYHA II-III) in the presence of a left ventricular ejection fraction (LVEF) $>50 \%$. Exclusion criteria included significant coronary artery disease which had not been revascularized; moderate or greater aortic or mitral valve disease; infiltrative, restrictive or hypertrophic myocardial disease; pericardial constriction; or significant right ventricular disease. The diagnosis of HFpEF was confirmed by the presence of an end-expiratory pulmonary capillary wedge pressure (PCWP) $\geq 15 \mathrm{mmHg}$ at rest or $\geq 25 \mathrm{mmHg}$ during symptom-limited exercise. Healthy subjects were recruited from the general community and were free from known cardiac or respiratory disease and displayed a normal exercise hemodynamic profile including a peak PCWP $<25 \mathrm{mmHg}$. The study was approved by the Institutional Ethics Review Committee, and all participants provided written informed consent.

\section{Sample preparation for mass spectrometry-based proteomics}

Frozen powdered heart tissue (10-20 mg) was weighed into tubes and lysed in $100 \mu \mathrm{L}$ of $4 \%$ sodium deoxycholate and $100 \mathrm{mM}$ Tris $\mathrm{HCl}$ ( $\mathrm{pH}$ adjusted to 8). Tissue lysates were then heated at $95^{\circ} \mathrm{C}$ and mixed at 1,000 rpm for 10 minutes using Thermomixer C (Eppendorf, Hamburg, Germany) before they were subjected to centrifugation at $18,000 \mathrm{xg}$ for 10 minutes at room temperature. Protein concentration of tissue lysates was determined by Bicinchoninic Acid (BCA) Protein Assay Kit (Pierce, Thermo Scientific, Waltham, MA, USA). $20 \mu \mathrm{g}$ of protein was subjected to reduction with TCEP and alkylated with chloroacetamide for 10 minutes at $95^{\circ} \mathrm{C}$ and $1,000 \mathrm{rpm}$ in the Thermomixer $\mathrm{C}$. Samples were then processed for trypsin digestion and subjected to a peptide cleanup as described previously ${ }^{49}$. Peptides were resuspended in $5 \%$ formic acid and stored at $4^{\circ} \mathrm{C}$ until acquired by LC-MS.

\section{Data-dependent mass spectrometry-based proteomics}

Using a Thermo Fisher Dionex Ultimate 3000 UHPLC, peptides in $5 \%$ (vol/vol) formic acid (500 ng) were directly injected onto a $50 \mathrm{~cm} \times 75 \mathrm{um}$ fused silica analytical column with a $\sim 10 \mu \mathrm{m}$ pulled tip containing C18 material (Dr. Maisch, Ammerbuch, Germany, $1.9 \mu \mathrm{m}, 130 \mathrm{~A}$ ), coupled online to a nanospray ESI source. Peptides were resolved over a gradient $5-40 \%$ acetonitrile over 120 min with a flow rate of $300 \mathrm{~nL}$ 
$\mathrm{min}^{-1}$. Peptides were ionized by electrospray ionization at $2.3 \mathrm{kV}$. Tandem mass spectrometry analysis was carried out on a Q-Exactive HFX mass spectrometer using HCD fragmentation (Thermo). The datadependent acquisition method acquired MS/MS spectra of the top 20 most abundant ions at any one point during the gradient. RAW MS data have been deposited to the ProteomeXchange Consortium via the PRIDE partner repository with the dataset identifier PXD026582 (Username:

reviewer_pxd026582@ebi.ac.uk, Password: oPiW26Wz).RAW data were analyzed using the quantitative proteomics software MaxQuant ${ }^{50}$ (version 1.6.3.4). Peptide and protein level identification were both set to a false discovery rate of $1 \%$ using a target-decoy based strategy. The database supplied to the search engine for peptide identifications contained both the mouse UniProt database downloaded on the 8th Nov 2018 and the MaxQuant contaminants database. Mass tolerance was set to $4.5 \mathrm{ppm}$ for precursor ions and MS/MS mass tolerance was 20 ppm. Enzyme specificity was set to trypsin (cleavage C-terminal to Lys and Arg) with a maximum of 2 missed cleavages permitted. Deamidation of Asn and GIn, oxidation of Met, pyro-Glu (with peptide N-term GIn) and protein N-terminal acetylation were set as variable modifications. Carbamidomethyl on Cys was searched as a fixed modification. We used the MaxLFQ algorithm for label-free quantitation, integrated into the MaxQuant environment ${ }^{51}$. Maxquant output was processed and statistical tests performed using the R software package (version 3.4.3) and processed data plotted using Tableau (version 10.0.2).

\section{Sample preparation for targeted metabolomics and non-targeted lipidomics}

Briefly, approximately 30-50 mg of powdered mice heart tissues were subjected to metabolite and lipid extraction prior to liquid chromatography-tandem mass spectrometry (LC-MS/MS) analysis. Targeted metabolite profiling used in this study was established using reference standards for each individual metabolite to determine MS multiple reaction-monitoring transitions, declustering potentials, collision energies, and chromatographic retention time, as described previously ${ }^{52}$. A stepwise extraction method was performed as previously described, using methanol/chloroform/water to efficiently extract lipids and metabolites from the tissues ${ }^{52,53}$.

\section{Targeted metabolomics}

For targeted metabolomic analysis, an LC-MS/MS system composed of a Shimadzu Nexera LC-30AD UHPLC (Shimadzu Corporation, Kyoto, Japan) system coupled to a QTRAP6500 mass spectrometer (AB Sciex, Foster City, CA, USA) was used to measure hydrophilic metabolites in positive and negative ionization modes. The polar metabolites in both positive and negative ionization mode were separated in hydrophilic interaction liquid chromatography (HILIC) mode using an Atlantis ${ }^{\circledR}$ HILIC column (Waters) and an XBridge ${ }^{\mathrm{TM}}$ Amide column (Waters), respectively, which allowed the separation of metabolites of different properties, both as previously described ${ }^{52,53}$. Sample analysis was conducted in a randomized sample order and each sample was run in duplicate. Data was acquired in the same batch on the same day. 
The analysis software Sciex OS 2.0 (ABSciex) was used for MRM Q1/Q3 peak integration of the raw data files (Analyst software, v.1.6.2; ABSciex). The peak area corresponds to the abundance of that metabolite; the abundance values were then normalized to their bookended pooled tissue extracts in the subsequent analysis, which were included after every 4 study samples in the sample queue, to account for temporal drift in instrument performance.

\section{Non-targeted lipidomics}

For analysis of lipid extracts from mice hearts, isotopically-labeled lipid standard mixture (SPLASH ${ }^{\mathrm{TM}}$ Lipidomix ${ }^{\circledR}$ Mass Spec Standard, Avanti Polar Lipids) was spiked into each sample, and the concentrations of identified lipid species from 14 major lipid classes included in the SPLASH were calculated relative to the known concentration of internal standards included in the SPLASH standard mixture.

Lipidomic profiling in this study used a Thermo Scientific ${ }^{\text {TM }}$ Vanquish $^{\text {TM }}$ UHPLC system and Thermo Scientific ${ }^{\text {TM }}$ Q Exactive ${ }^{T M} \mathrm{HF}$ hybrid quadrupole-Orbitrap ${ }^{\mathrm{TM}}$ mass spectrometer, with lipid separation performed on a Thermo Scientific ${ }^{T M}$ Acclaim ${ }^{T M} \mathrm{C} 30$ column $(2.1 \times 150 \mathrm{~mm}, 2.6 \mu \mathrm{m})$, operated at $45^{\circ} \mathrm{C}$, flow rate of $260 \mu \mathrm{L} / \mathrm{min}$. Lipid extracts from the mice hearts were analyzed using the LC conditions and MS instrument conditions in both positive and negative ion modes, as described previously ${ }^{54}$. Lipid Search 4.1 software was used to align and process each acquired raw file's search results, as well as to identify the lipid species present in the heart extracts ${ }^{55}$.

\section{Tissue homogenization and lysis for enzymatic activity assay}

For the determination of HMGCS2 activity in mice, a total of 2-3 mouse heart tissues were combined for each assay ( $n=5-7$ pooled heart tissues assayed for controls and $n=6-8$ pooled heart tissues assayed for HFpEF mice). Heart extracts were prepared by lysis in $500 \mu \mathrm{L}$ of ice-cold mammalian cell lysis extraction mixture containing protease inhibitor cocktail solutions (MCL1; Sigma-Aldrich). Tissues were homogenized by Qiagen TissueLyser LT ( $50 \mathrm{cycle} / \mathrm{sec})$ for 2 minutes. Particulate matter was then separated by centrifugation for 10 minutes at $4{ }^{\circ} \mathrm{C}$ at $11,000 \times \mathrm{g}$. The resulting clear supernatant was collected for incubation. Human myocardial tissues were subjected to the same tissue homogenization procedures for extract preparation for mice.

\section{HMGCS2 enzymatic activity}

The protein content of the lysate mixture was determined using the Bicinchonic Acid (BCA) Protein Assay Kit (Pierce Biotechnology, Rockford, IL, USA) according to the manufacturer's instructions. HMGCS2 activity assay in mouse and human heart tissue was carried out with modification as described previously ${ }^{74,75}$. HMGCS2 activity was measured based on the incorporation of isotope-labeled [1,2${ }^{13} \mathrm{C}_{2}$ ] acetyl-CoA to isotope-labeled acetoacetate at $\mathrm{pH} 8$ and temperature set to $37^{\circ} \mathrm{C}$. To $250 \mu \mathrm{L}$ of reaction mixture containing $50 \mathrm{mM}$ Tris, $0.01 \mathrm{mM}$ of acetoacetyl-CoA, $1 \mathrm{mM}$ of $\left[1,2-{ }^{13} \mathrm{C}_{2}\right]$ acetyl-CoA (Sigma, 658650-5MG), $150 \mu \mathrm{L}(0.225 \mathrm{mg} / \mathrm{mL})$ of the tissue homogenate was added. Reaction proceeded 
at $37^{\circ} \mathrm{C}$ for $5,10,20,30,45,60,90,120,150,180$, and 210 minutes, with removal of $30 \mu \mathrm{L}$ of aliquots of the reaction mixture, and the enzymatic reaction was stopped immediately by adding $5 \mu \mathrm{L}$ of $5 \mathrm{M}$ perchloric acid starting at time $=0$ and the indicated time points. Measurement of ${ }^{13} \mathrm{C}$-labeling in intermediates of the ketogenic pathway (acetoacetyl-CoA and HMG-CoA) was made using liquidchromatography tandem mass spectrometry.

To test for any non-enzymatic production of isotope-labeled acetoacetyl-CoA, HMG-CoA and acetoacetate, which would interfere with the HMGCS2 activity assay, samples comprising the lysis buffer plus the other chemicals, but no tissue, were incubated and analyzed exactly the way as normal tissue samples.

Calibration against analytical standards was carried out using labeled acetyl-CoA, unlabeled acetoacetylCoA, HMG-CoA and acetoacetate in the concentration ranges of $0.03-100 \mu \mathrm{mol} / \mathrm{l}$. All standards were not exposed to the incubation but were added with the same amount of perchloric acid, followed by similar sample preparation steps to provide as similar as possible sample composition in comparison to the incubated tissue samples. Concentration values of ${ }^{13} \mathrm{C}_{4}$-AcAc were obtained in the unit of $\mu \mathrm{mol} / \mathrm{I}$. These values were divided by the incubation time (hrs), and the protein concentrations, respectively, in the unit $\mathrm{mg} / \mathrm{l}$. The resulting enzymatic activity values were expressed in total ${ }^{13} \mathrm{C}_{4}$-AcAc generated $\mu \mathrm{mol} / \mathrm{mg}$ protein/hr, which were transformed to the final unit nmol.mg $\mathrm{p}^{-1} . \mathrm{hr}^{-1}$ for ease of interpretation.

\section{Immunoblotting}

Protein concentration of the resultant supernatant from the enzymatic assay was determined using the Bicinchonic Acid (BCA) Protein Assay Kit (Pierce), following manufacturer's instructions. $40 \mu \mathrm{g}$ of total protein of heart tissue lysates were heat denatured with $4 \times$ Laemmli sample buffer (Bio-Rad, Hercules, CA) supplemented with $10 \%$-mercaptoethanol (Sigma) at $95^{\circ} \mathrm{C}$ for 5 minutes, and the proteins were resolved on 4-15\% Mini-PROTEAN ${ }^{\circledR}$ TGX $^{\text {TM }}$ Precast Gel (Bio-Rad), and transferred to a PVDF membrane (Thermo Fisher Scientific). Blocking of the membrane was performed using $5 \%$ non-fat milk in Trisbuffered saline (TBS) containing $0.1 \%$ Tween-20 for 1 hour. The membrane was then incubated with a primary antibody at $4^{\circ} \mathrm{C}$ overnight. Following three subsequent washings in TBST, the membrane was then probed with secondary horseradish peroxidase conjugated antibody [anti-rabbit (P0448, Dako, Carpinteria, CA, USA) or anti-mouse (P0447, Dako, Carpinteria, CA, USA)] for 45 minutes. The blots were then visualized using ImageQuant LAS 4010 digital imaging system (GE Healthcare). The protein levels were normalized to actin and compared to the control group. Quantitation was completed with Image J. Each experiment was completed with $n \geq 2$. The primary antibodies and dilutions probed for the target proteins are shown in the table below. 


\begin{tabular}{|c|c|c|c|}
\hline Antibody & Dilution & Secondary & Supplier \\
\hline HMGCS2 (ab137043) & $1: 1000$ & Rabbit & Abcam \\
\hline Acetylated-Lysine (Ac-K2-100) (9814S) & $1: 1000$ & Rabbit & Cell Signaling \\
\hline Actin (ab8226) & $1: 1000$ & Mouse & Abcam \\
\hline
\end{tabular}

\section{Statistical analysis}

Absolute values of fold changes among groups were calculated for metabolomic and proteomic data and were Log2 transformed using R (version 3.6.1). Statistical significance was determined using unpaired nonparametric t-test. A $P$-value $\leq 0.05$ was considered statistically significant for the two experimental groups. Protein outputs were corrected for multiple testing using the Benjamini-Hochberg correction, with significance being set at $P \leq 0.05$ at an FDR of $5 \%$ and plotted using Tableau or GraphPad Prism software 8.0. Transcardiac ketone body gradients were compared using Welch Two-Sample T-test, using $\mathrm{R}$ version 4.0.2.

\section{Declarations}

Acknowledgements:

We would like to acknowledge Ms Shashwati Dutta for statistical analysis, Dr Lake-Ee Quek for help with the stable-isotope experiments, and Sydney Mass Spectrometry for use of instruments. This work was supported by the Heart Research Institute (YCK, JFOS); Sydney Medical School Foundation [JFOS]; NSW Health [JFOS DOH1003; DOH1006]; and the National Heart Foundation [JFOS NHF104853]. ML is a Cancer Institute New South Wales Future Research Leader Fellow.

\section{Author contributions:}

YCK and JFOS conceived the study and wrote the manuscript. YCK performed the murine work, metabolomics, stable-isotope labelled experiments, lipidomics, Western blot, immunoprecipitation, and analyses. YC performed murine experiments and analyses. AYB performed murine experiments. SL oversaw the human myocardial tissue analysis. AJL oversaw murine experiments, analyses, and wrote the manuscript. DMK performed and analysed the human invasive haemodynamic and transcoronary gradient experiments. ML performed and analysed the proteomics. 
Competing Interest Declaration:

_The authors have no competing interests to declare.

\section{References}

1 Gladden, J. D., Chaanine, A. H. \& Redfield, M. M. Heart Failure with Preserved Ejection Fraction. Annu. Rev. Med. 69, 65-79, doi:10.1146/annurev-med-041316-090654 (2018).

2 Wang, Z. et al. Specific metabolic rates of major organs and tissues across adulthood: evaluation by mechanistic model of resting energy expenditure. Am. J. Clin. Nutr. 92, 1369-1377, doi:10.3945/ajen.2010.29885 (2010).

3 Ashrafian, H., Frenneaux, M. P. \& Opie, L. H. Metabolic mechanisms in heart failure. Circulation 116, 434-448, doi:10.1161/CIRCULATIONAHA.107.702795 (2007).

4 Ferrannini, E., Mark, M. \& Mayoux, E. CV Protection in the EMPA-REg OUTCOME Trial: A "Thrifty Substrate" Hypothesis. Diabetes Care 39, 1108-1114, doi:10.2337/dc16-0330 (2016).

$5 \quad$ Kashiwaya, Y. et al. A ketone ester diet increases brain malonyl-CoA and Uncoupling proteins 4 and 5 while decreasing food intake in the normal Wistar Rat. J. Biol. Chem. 285, 25950-25956, doi:10.1074/jbc.M110.138198 (2010).

6 Sato, K. et al. Insulin, ketone bodies, and mitochondrial energy transduction. FASEB J. 9, 651-658, doi:10.1096/fasebj.9.8.7768357 (1995).

7 Veech, R. L. Ketone esters increase brown fat in mice and overcome insulin resistance in other tissues in the rat. Ann. N. Y. Acad. Sci. 1302, 42-48, doi:10.1111/nyas.12222 (2013).

8 Nielsen, R. et al. Cardiovascular Effects of Treatment With the Ketone Body 3-Hydroxybutyrate in Chronic Heart Failure Patients. Circulation 139, 2129-2141, doi:10.1161/CIRCULATIONAHA.118.036459 (2019).

9 Zhang, Y. et al. Mitochondrial pyruvate carriers are required for myocardial stress adaptation. Nat. Metab. 2, 1248-1264, doi:10.1038/s42255-020-00288-1 (2020).

10 Deng, Y. et al. Targeting Mitochondria-Inflammation Circuit by $\beta$-Hydroxybutyrate Mitigates HFpEF. Circ. Res. 128, 232-245, doi:10.1161/circresaha.120.317933 (2021).

11 Borlaug, B. A., Nishimura, R. A., Sorajja, P., Lam, C. S. P. \& Redfield, M. M. Exercise hemodynamics enhance diagnosis of early heart failure with preserved ejection fraction. Circ. Heart Fail. 3, 588-595, doi:10.1161/CIRCHEARTFAILURE.109.930701 (2010).

12 Eisman, A. S. et al. Pulmonary Capillary Wedge Pressure Patterns During Exercise Predict Exercise Capacity and Incident Heart Failure. Circ. Heart Fail. 11, e004750, 
doi:10.1161/CIRCHEARTFAILURE.117.004750 (2018).

13 Schiattarella, G. G. et al. Nitrosative stress drives heart failure with preserved ejection fraction. Nature 568, 351-356, doi:10.1038/s41586-019-1100-z (2019).

14 Abel, E. D. Glucose transport in the heart. Front. Biosci. 9, 201-215, doi:10.2741/1216 (2004).

15 Pettersen, I. K. N. et al. Upregulated PDK4 expression is a sensitive marker of increased fatty acid oxidation. Mitochondrion 49, 97-110, doi:10.1016/j.mito.2019.07.009 (2019).

16 Longo, N., Frigeni, M. \& Pasquali, M. Carnitine transport and fatty acid oxidation. Biochim. Biophys. Acta 1863, 2422-2435, doi:10.1016/j.bbamcr.2016.01.023 (2016).

17 Neubauer, S. et al. Myocardial phosphocreatine-to-ATP ratio is a predictor of mortality in patients with dilated cardiomyopathy. Circulation 96, 2190-2196, doi:10.1161/01.cir.96.7.2190 (1997).

18 Phan, T. T. et al. Heart failure with preserved ejection fraction is characterized by dynamic impairment of active relaxation and contraction of the left ventricle on exercise and associated with myocardial energy deficiency. J. Am. Coll. Cardiol. 54, 402-409, doi:10.1016/j.jacc.2009.05.012 (2009).

19 Shimazu, T. et al. SIRT3 deacetylates mitochondrial 3-hydroxy-3-methylglutaryl CoA synthase 2 and regulates ketone body production. Cell Metab. 12, 654-661, doi:10.1016/j.cmet.2010.11.003 (2010).

20 Nogueiras, R. et al. Sirtuin 1 and sirtuin 3: physiological modulators of metabolism. Physiol. Rev. (2012).

21 Bach, A. Oxaloacetate deficiency in MCT-induced ketogenesis. Arch. Int. Physiol. Biochim. 86, 1133-1142, doi:10.3109/13813457809055968 (1978).

22 Ingwall, J. S., Friedrich, J. \& Nascimben, L. Does Decreased Energy Supply Contribute to Heart Failure? The Role of the Creatine Kinase System. Developments in Cardiovascular Medicine, 231-239, doi:10.1007/978-1-4613-1237-6_18 (1995).

23 Schulze, P. C. \& Wu, J. M. F. Ketone bodies for the starving heart. Nat. Metab. 2, 1183-1185, doi:10.1038/s42255-020-00310-6 (2020).

24 Puchalska, P. \& Crawford, P. A. Multi-dimensional Roles of Ketone Bodies in Fuel Metabolism, Signaling, and Therapeutics. Cell Metab. 25, 262-284, doi:10.1016/j.cmet.2016.12.022 (2017).

25 Cotter, D. G., d'Avignon, D. A., Wentz, A. E., Weber, M. L. \& Crawford, P. A. Obligate role for ketone body oxidation in neonatal metabolic homeostasis. J. Biol. Chem. 286, 6902-6910, doi:10.1074/jbc.M110.192369 (2011).

26 Bing, R. J. The metabolism of the heart. Trans. Am. Coll. Cardiol. 5, 8-14 (1955). 
27 Garland, P. B., Newsholme, E. A. \& Randle, P. J. Effect of fatty acids, ketone bodies, diabetes and starvation on pyruvate metabolism in rat heart and diaphragm muscle. Nature 195, 381-383, doi:10.1038/195381a0 (1962).

28 Hasselbaink, D. M., Glatz, J. F. C., Luiken, J. J. F. P., Roemen, T. H. M. \& Van der Vusse, G. J. Ketone bodies disturb fatty acid handling in isolated cardiomyocytes derived from control and diabetic rats. Biochem. J. 371, 753-760, doi:10.1042/BJ20021617 (2003).

29 Jeffrey, F. M., Diczku, V., Sherry, A. D. \& Malloy, C. R. Substrate selection in the isolated working rat heart: effects of reperfusion, afterload, and concentration. Basic Res. Cardiol. 90, 388-396, doi:10.1007/BF00788500 (1995).

30 Tardif, A. et al. Chronic exposure to beta-hydroxybutyrate impairs insulin action in primary cultures of adult cardiomyocytes. Am. J. Physiol. Endocrinol. Metab. 281, E1205-1212, doi:10.1152/ajpendo.2001.281.6.E1205 (2001).

31 Yan, J. et al. Increased glucose uptake and oxidation in mouse hearts prevent high fatty acid oxidation but cause cardiac dysfunction in diet-induced obesity. Circulation 119, 2818-2828, doi:10.1161/CIRCULATIONAHA.108.832915 (2009).

32 Goldman, D. S. Studies on the fatty acid oxidizing system of animal tissues. VII. The beta-ketoacyl coenzyme A cleavage enzyme. J. Biol. Chem. 208, 345-357 (1954).

33 Shukla, S. K. et al. HMGCS2 is a key ketogenic enzyme potentially involved in type 1 diabetes with high cardiovascular risk. Sci. Rep. 7, 4590, doi:10.1038/s41598-017-04469-z (2017).

34 Cook, G. A., Lavrentyev, E. N., Pham, K. \& Park, E. A. Streptozotocin diabetes increases mRNA expression of ketogenic enzymes in the rat heart. Biochim. Biophys. Acta Gen. Subj. 1861, 307-312, doi:10.1016/j.bbagen.2016.11.012 (2017).

35 Walker, M. A. \& Tian, R. NAD $(\mathrm{H})$ in mitochondrial energy transduction: implications for health and disease. Curr. Opin. Physiol. 3, 101-109, doi:10.1016/j.cophys.2018.03.011 (2018).

36 Sundaresan, N. R. et al. Sirt3 blocks the cardiac hypertrophic response by augmenting Foxo3adependent antioxidant defense mechanisms in mice. J. Clin. Invest. 119, 2758-2771, doi:10.1172/JCl39162 (2009).

37 Koentges, C. et al. SIRT3 deficiency impairs mitochondrial and contractile function in the heart. Basic Res. Cardiol. 110, 36, doi:10.1007/s00395-015-0493-6 (2015).

38 Pillai, V. B., Sundaresan, N. R., Jeevanandam, V. \& Gupta, M. P. Mitochondrial SIRT3 and heart disease. Cardiovasc. Res. 88, 250-256, doi:10.1093/cvr/cvq250 (2010). 
39 Murashige, D. et al. Comprehensive quantification of fuel use by the failing and nonfailing human heart. Science 370, 364-368, doi:10.1126/science.abc8861 (2020).

40 Voros, G. et al. Increased Cardiac Uptake of Ketone Bodies and Free Fatty Acids in Human Heart Failure and Hypertrophic Left Ventricular Remodeling. Circ. Heart Fail. 11, e004953, doi:10.1161/CIRCHEARTFAILURE.118.004953 (2018).

41 McCommis, K. S. et al. Nutritional modulation of heart failure in mitochondrial pyruvate carrierdeficient mice. Nat. Metab. 2, 1232-1247, doi:10.1038/s42255-020-00296-1 (2020).

42 Karwi, Q. G., Uddin, G. M., Ho, K. L. \& Lopaschuk, G. D. Loss of Metabolic Flexibility in the Failing Heart. Front. Cardiovasc. Med. 5, 68, doi:10.3389/fcvm.2018.00068 (2018).

43. Schnelle, M. et al. Echocardiographic evaluation of diastolic function in mouse models of heart disease. J. Mol. Cell. Cardiol. 114, 20-28 (2018).

44. van Dijk, T. H. et al. A novel approach to monitor glucose metabolism using stable isotopically labelled glucose in longitudinal studies in mice. Lab. Anim. 47, 79-88 (2013).

45. Matthews, D. R. et al. Homeostasis model assessment: insulin resistance and beta-cell function from fasting plasma glucose and insulin concentrations in man. Diabetologia 28, 412-419 (1985).

46. Lal, S. et al. Best Practice BioBanking of Human Heart Tissue. Biophys. Rev. 7, 399-406 (2015).

47. Mamidi, R. et al. Dose-Dependent Effects of the Myosin Activator Omecamtiv Mecarbil on CrossBridge Behavior and Force Generation in Failing Human Myocardium. Circ. Heart Fail. 10, (2017).

48. Sequeira, V. et al. ADP-stimulated contraction: A predictor of thin-filament activation in cardiac disease. Proc. Natl. Acad. Sci. U. S. A. 112, E7003-12 (2015).

49. Harney, D. J. et al. Proteomic Analysis of Human Plasma during Intermittent Fasting. J. Proteome Res. 18, 2228-2240 (2019).

50. Cox, J. \& Mann, M. MaxQuant enables high peptide identification rates, individualized p.p.b.-range mass accuracies and proteome-wide protein quantification. Nat. Biotechnol. 26, 1367-1372 (2008).

51. Cox, J. et al. Accurate proteome-wide label-free quantification by delayed normalization and maximal peptide ratio extraction, termed MaxLFQ. Mol. Cell. Proteomics 13, 2513-2526 (2014).

52. Effect of chronic exercise in healthy young male adults: a metabolomic analysis.

Yen Chin Koay, Kelly Stanton, Vivian Kienzle, Mengbo Li, Jean Yang, David S. Celermajer, John F. O’Sullivan. Cardiovasc. Res. 2021 Jan 21;117(2):613-622. 
53. Koay, Y. C. et al. Ingestion of resistant starch by mice markedly increases microbiome-derived metabolites. FASEB J. 33, 8033-8042 (2019).

54. Kiyonami, R., Peake, D. A., Liu, X. \& Huang, Y. in LIPID MAPS Annual Meeting. 12-13.

55. Peake, D. A., Kiyonami, R., Yokoi, Y., Fukamachi, Y. \& Huang, Y. in ASMS annual meeting, Saint Louis, MO, 31st May-4th June.

\section{Figures}

(a)

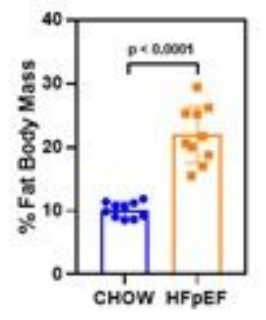

(d)

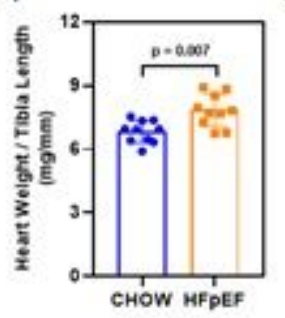

(b)

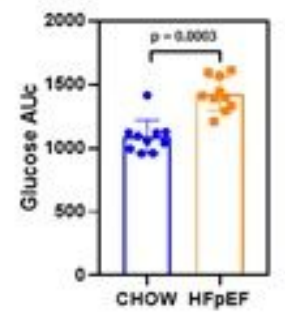

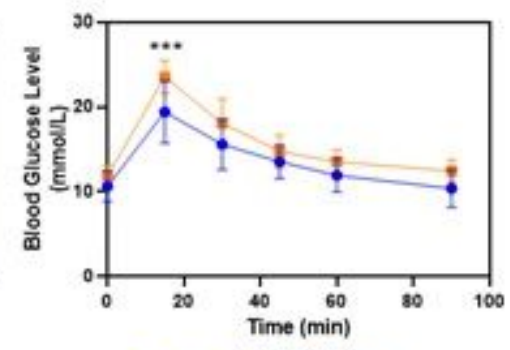

- CHOW - FFDEF

(g)

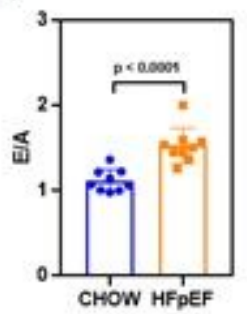

(f)

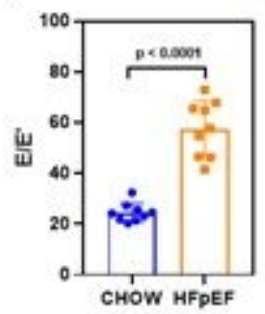

(c)

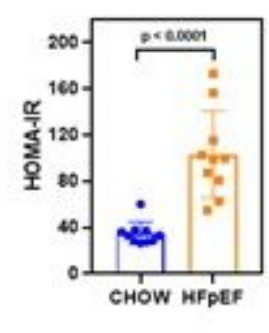

(h)

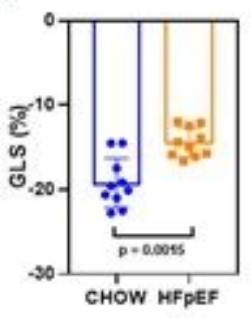

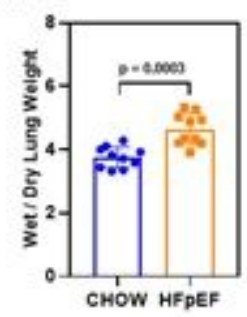

(i)

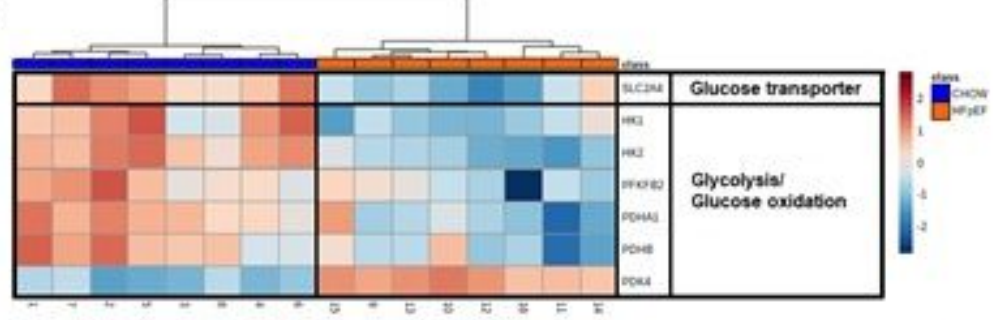

(j)

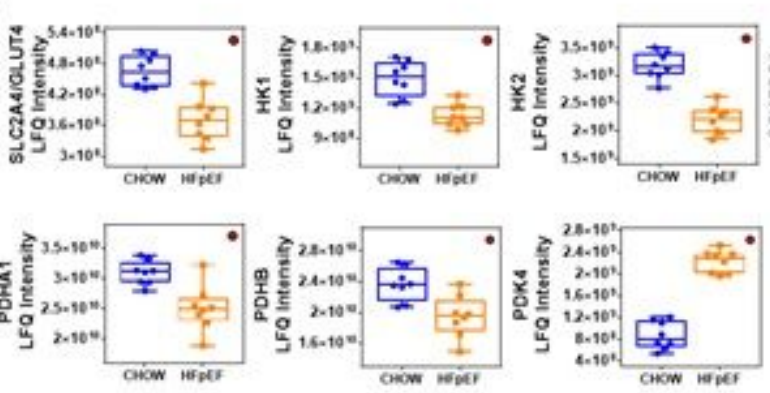

(k)

HFpEF Myocardium

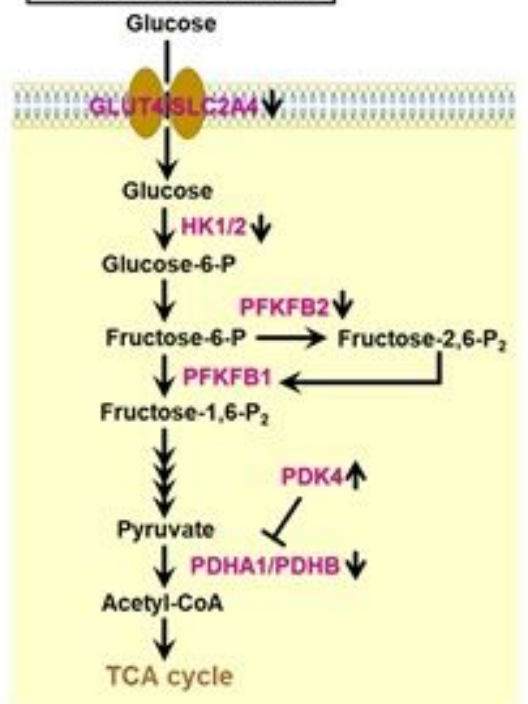


HFD and L-NAME administration led to obesity, glucose intolerance, insulin resistance and features of HFpEF, indicated by (a) fat mass, (b) increased glucose excursion, (c) increased HOMA-IR, (d) heart weight/tibia length, (e) increased transmittal doppler $E / A$ wave ratio, $(f)$ increased transmitral $E$ to tissue doppler E' (E/E') ratio, (g) impaired global longitudinal strain; (h) pulmonary oedema indicated by increased wet/dry lung weight. (i) Heatmap showing decreased expression of glucose transporter and glycolytic enzymes in HFpEF myocardium, where red indicates high protein abundance and blue indicates low protein abundance. Each column represents an individual mouse. (j) Box-and-whisker plots with individual dot points illustrating significant reductions of several rate-limiting enzymes critical for the regulation of glycolysis and glucose oxidation, with upregulation of PDK4 that inhibits the pyruvate dehydrogenase complex leading to metabolic inflexibility. Filled circles represent statistical significance $(p<0.05$ or $5 \%$ FDR) comparing HFpEF vs chow mice, as indicated by the legend. ( $k$ ) Schematic summarising the glycolytic pathway changes. Abbreviations: GLUT4: glucose transporter type 4;HK1 and 2: hexokinase 1 and 2; PFKFB2: phosphofructokinase 2; PFKFB1: phosphofructokinase 1; PDK4: pyruvate dehydrogenase kinase 4; PDHa and PDHb: pyruvate dehydrogenase $a$ and b; LFQ: label-free quantitation intensity. 

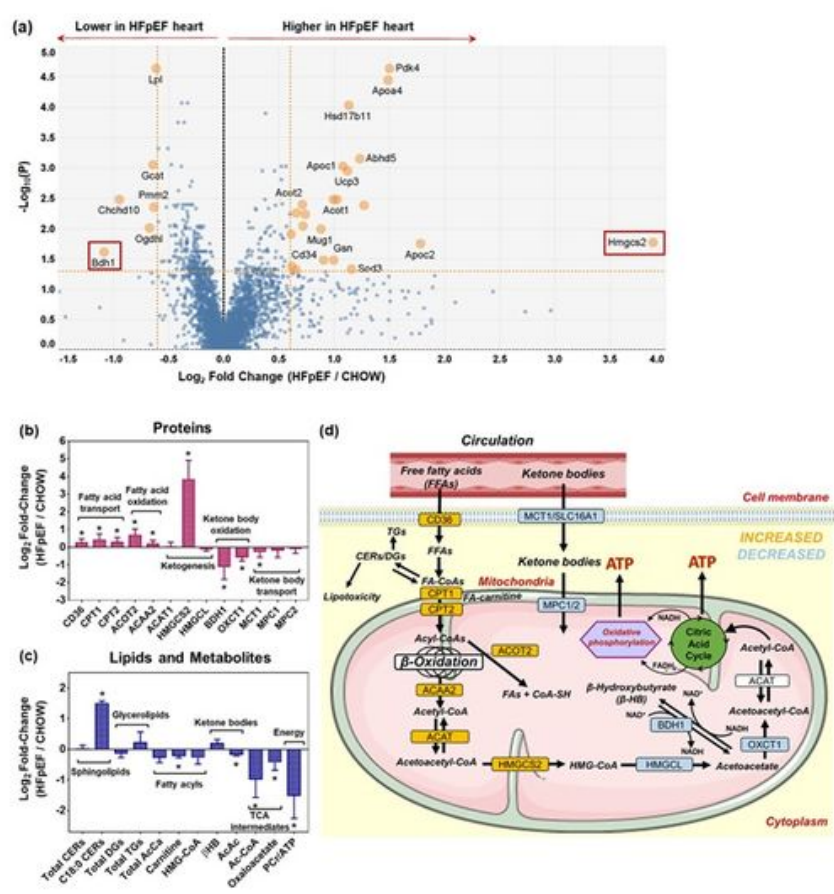

Human HFpEF: \% Cardiac Uptake

$\begin{array}{ll}\text { (e) BHydroxybutyrate } & \text { (f) Acetoacetate }\end{array}$

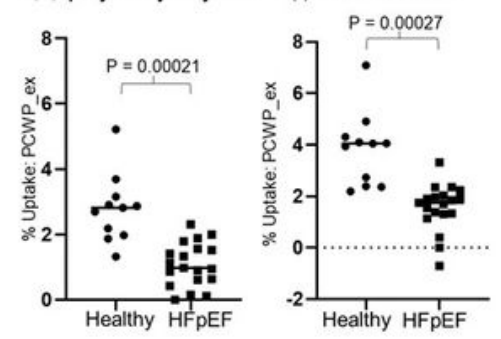

Human HFpEF: \% Cardiac Uptake $\beta \mathrm{Hb}$ vs AcAc

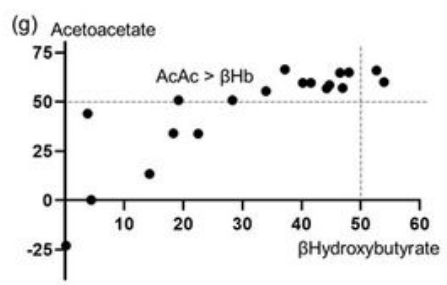

\section{Figure 2}

(a) Volcano plot depicting differentially-abundant proteins in HFpEF vs control myocardium. The X-axis displays log2 fold change and the y-axis displays - log10 Benjamini-Hochberg-adjusted $p$-values. Dashed horizontal line shows p-value cutoff $(<0.05)$, and the two vertical dashed lines represent the fold change cut-off of 1.5 ( 0.6 on log2 scale). (b) Bar plot illustrating changes in proteins involved in fatty acid transport, ketogenesis, and ketone oxidation in HFpEF vs control myocardium with log2 fold change on 
the y-axis, significance indicated with *. (c) Bar plot illustrating changes in substrates and products of the fatty acid transport, fatty acid oxidation, ketogenic, and ketone oxidation pathways in HFpEF vs control myocardium with log2 fold change on the $y$-axis, significance indicated with *. ${ }^{*} \mathrm{P} \leq 0.05$ (murine experiments, $n=8$ mice/group) (d) Schematic summarising the changes in ketogenic and lipid oxidation pathways with subcellular location indicated. HMGCS2 is seen as an inflection point between increases and decreases in pathway intermediates; proteins indicated in yellow denote upregulation and blue downregulation in the HFpEF heart. The ratios of percentage cardiac uptake (Arterial - coronary sinus concentrations / Arterial concentrations * 100): PCWP with exercise of (e) $\beta$-Hydroxybutyrate and (f) acetoacetate were significantly decreased in HFpEF hearts compared to controls. A positive number indicates net uptake by the heart, whereas a negative number indicates net secretion. (g) Percentage cardiac uptake assessment illustrated more acetoacetate ( $\mathrm{Y}$-axis) uptake than $\beta$-hydroxybutyrate ( $\mathrm{X}$-axis) in human HFpEF hearts (human transcardiac gradients, $n=13$ controls, $n=19$ HFpEF patients). Abbreviations: CD36: cluster of differentiation; CPT1: carnitine palmitoyltransferase l; CPT2: carnitine palmitoyltransferase 2; ACOT2: acyl-coenzyme A thioesterase 2; ACAA2: acetyl-coenzyme A acyltransferase 2; ACAT: acetyl-CoA acetyltransferase; HMGCS2: 3-hydroxy-3-methylglutaryl-CoA synthase 2; HMGCL: 3-hydroxymethyl-3-methylglutaryl-CoA lyase; $\mathrm{BDH1}$ : 3-hydroxybutyrate dehydrogenase; OXCT1: 3-oxoacid CoA-transferase 1; MCT1: monocarboxylate transporter 1; MPC: mitochondrial pyruvate carrier; PCWP: pulmonary capillary wedge pressure. 
(a)

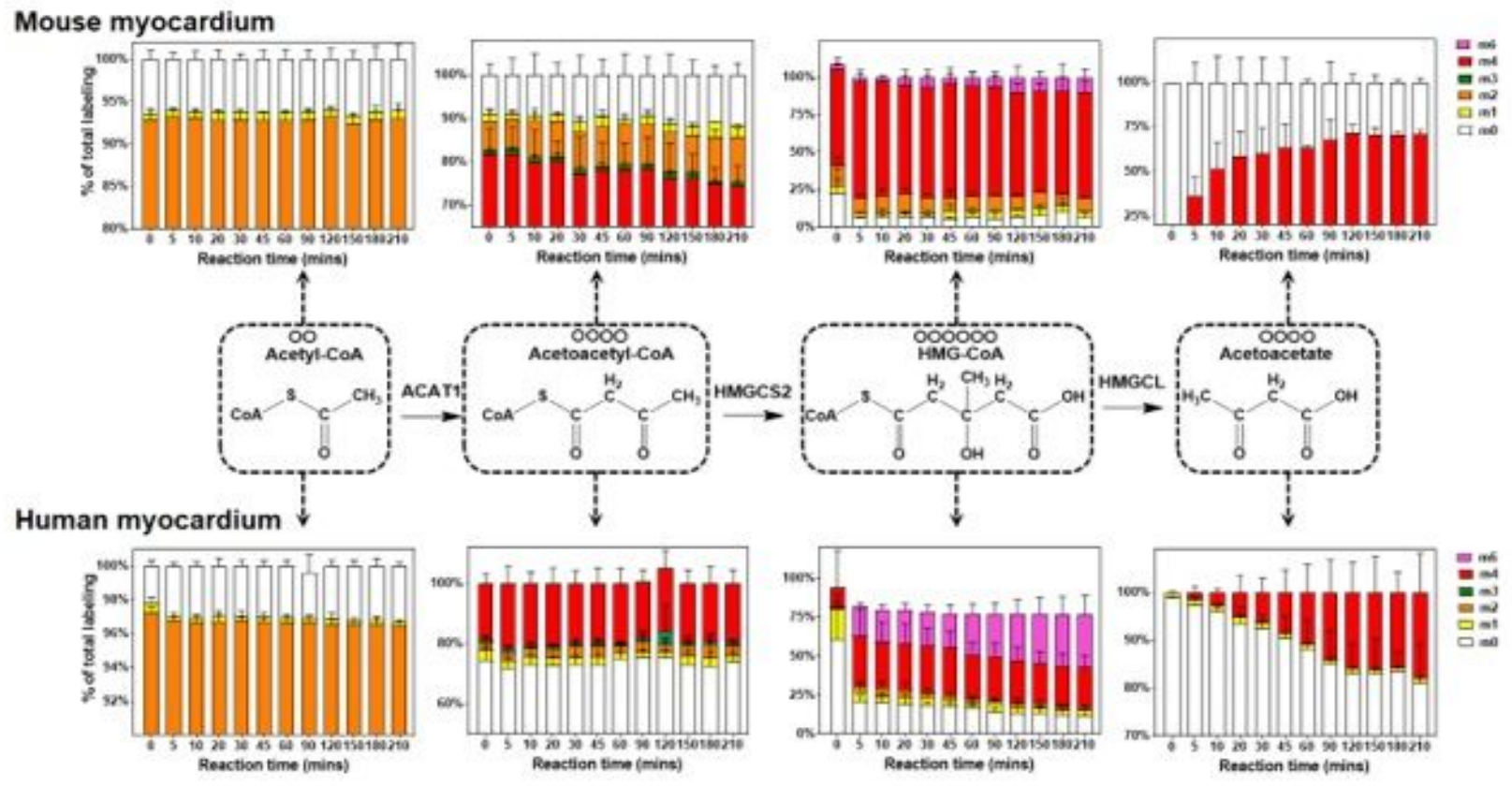

(b)
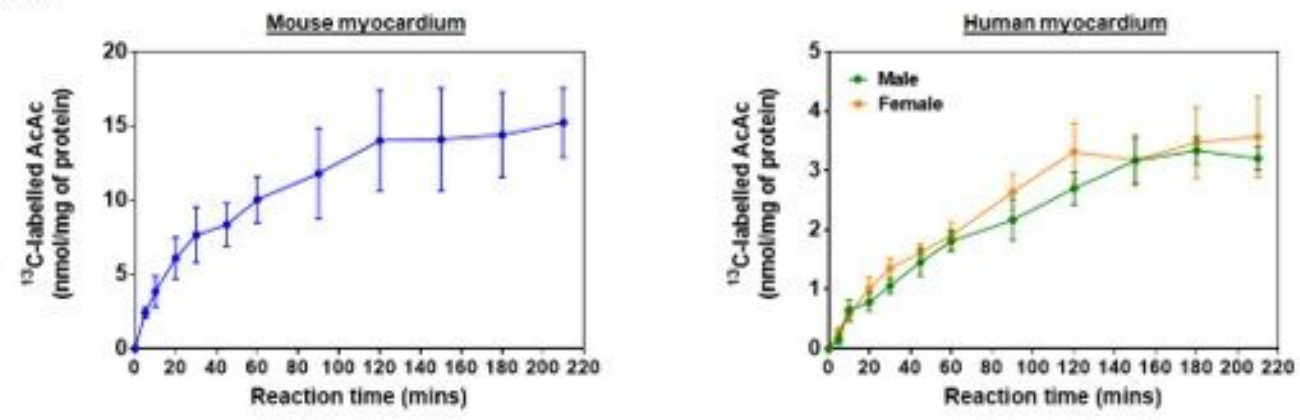

Figure 3

Stable-isotope tracing of myocardial ketogenesis. a) Corresponding $13 \mathrm{C}$ labelled ketogenesis intermediates from [1,2-13C2]-acetyl-CoA in murine $(n=3)$ and human $(n=7)$ myocardium. The circles above each metabolite name denote the number of labelled carbons considered for that metabolite. Isotopologues of each substrate are denoted by the number of $13 \mathrm{C}$-labelled carbons: for instance, $\mathrm{m} 0 \mathrm{has}$ no labelled carbons (i.e. is unlabelled), $\mathrm{m} 1$ has one 13C-labelled carbon, $\mathrm{m} 2$ has two 13C-labelled carbons, etc. The data are presented as means \pm SD (error bars) for individual isotopologues. (b) 13C4labelled acetoacetate measured at select time intervals in murine $(n=3)$ and human $(n=7)$ myocardium. 

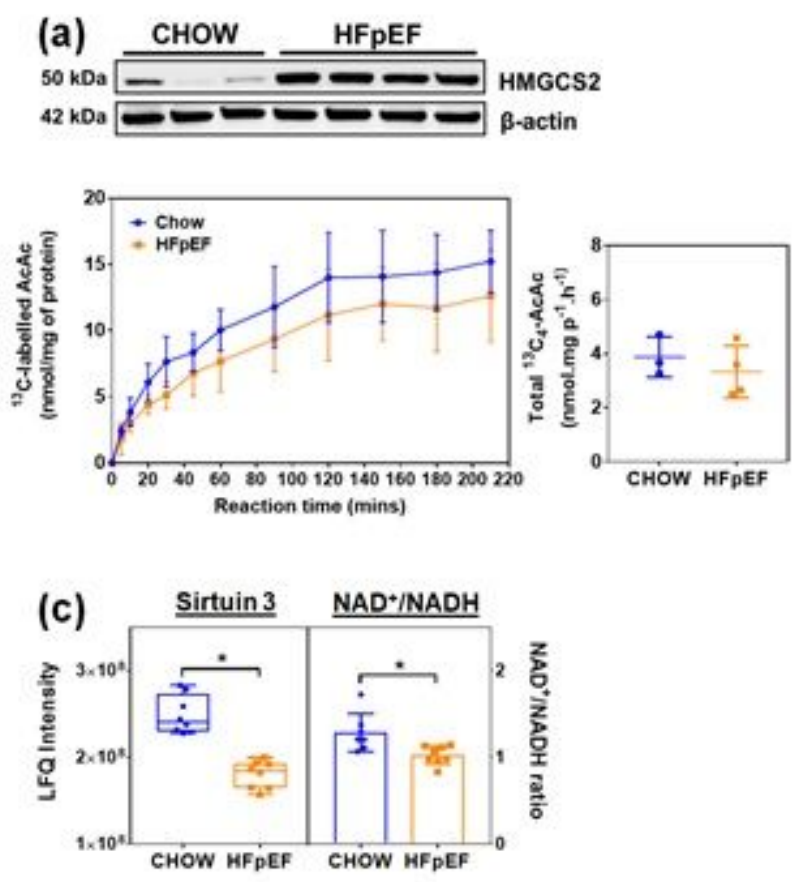
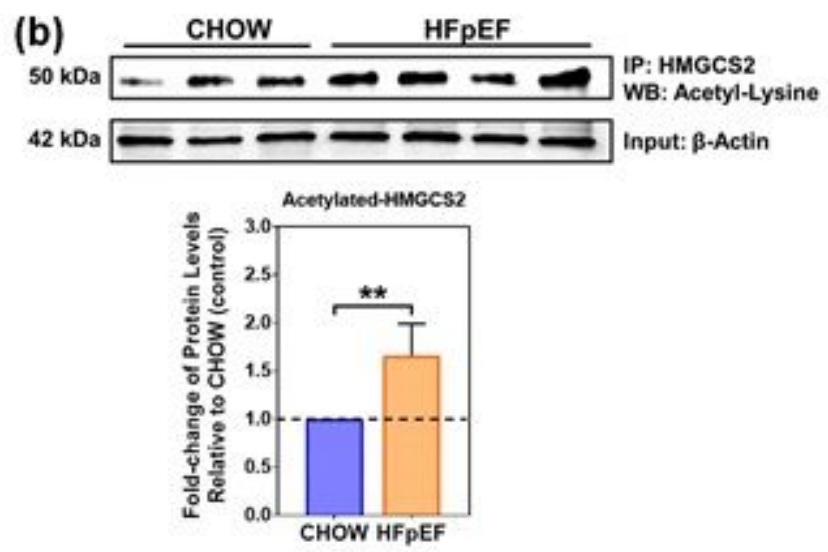

(d)

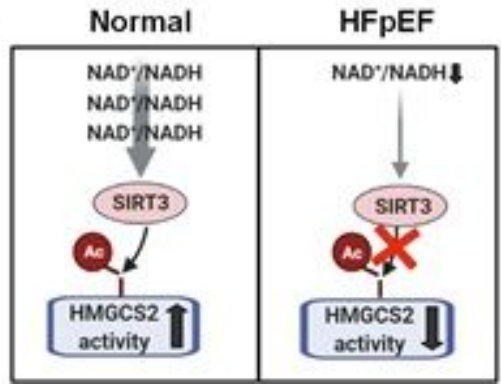

Figure 4

NAD+ depletion and reduced myocardial ketogenic activity. (a) Total 13C4-labelled acetoacetate from labeled [1,2-13C2]-acetyl-CoA was not significantly different in HFpEF vs control myocardium in mice $(\mathrm{n}=$ 3 mice for chow; $\mathrm{n}=4$ mice for HFpEF). (b) Immunoprecipitated HMGCS2 from HFpEF myocardium revealed greater acetylation ( $n=3$ mice for chow; $n=4$ mice for HFpEF). (c) The bar graph shows the abundance of Sirtuin 3 protein and ratios of NAD+/NADH in hearts from HFpEF mice over controls $(n=8$ mice/group). (d) Schematic proposing depletion of NAD+ causing decreased sirtuin 3 that impairs deacetylation, leading to decreased HMGCS2 activity. Statistical significance between chow versus HFpEF is indicated by *, $\mathrm{P} \leq 0.05$ and **, $\mathrm{P} \leq 0.01$. Abbreviations: HMGCS2: 3-hydroxy-3-methylglutaryl-CoA synthase 2; NAD+: oxidized form of nicotinamide adenine dinucleotide; NADH: reduced form of nicotinamide adenine dinucleotide.

\section{Supplementary Files}

This is a list of supplementary files associated with this preprint. Click to download.

- ExtendedDataFiguresandTable.pdf 